\title{
Fundamentos filosóficos y perspectivas actuales de un abordaje humeano al problema de las otras mentes
}

\author{
LEANDRO GUERRERO \\ Universidad de Buenos Aires \\ leandro_g00@hotmail.com
}

\begin{abstract}
Resumen: En este trabajo se explora el "problema de las otras mentes" desde una perspectiva humeana, con la intención de concebir una alternativa anticartesiana tanto en el nivel teórico como en el metateórico. Para ello, se examinan brevemente algunas de las características más importantes de la teoría humeana de la subjetividad, sistemáticamente desatendidas por la mayoría de los intérpretes: la preponderancia de la dimensión pasional (de raigambre intersubjetiva) en la formación gradual de la subjetividad y el papel de la simpatía en ese proceso. Además, se sostiene la posibilidad de elaborar una perspectiva anticartesiana sobre la base de una inversión metateórica ineludible, un verdadero giro práctico que tiene lugar dentro de la propuesta humeana, el cual implica hacer un marcado hincapié en la primacía del aspecto práctico-intersubjetivo de la subjetividad por encima del aspecto teórico-individualista.
\end{abstract}

Palabras clave: sujeto, alter ego, simpatía, intersubjetividad, praxis

\begin{abstract}
This paper explores the "other minds problem" from a Humean perspective, with the objective of devising an anti-Cartesian alternative, both theoretical and meta-theoretical. In order to do this, the paper briefly examines some of the most important features of the Humean theory of the self systematically neglected by most interpreters: the prevalence of the passional dimension (intersubjectively rooted) in the gradual formation of the self, and the role of sympathy in that process. We hold the possibility of developing an anti-Cartesian perspective on the basis of an unavoidable meta-theoretical reversal, a genuine practical turn that takes place within the Humean proposal, and which involves a greater emphasis on the primacy of the practicalintersubjective aspect of the self over the theoretical-individualistic one.

Key words: self, alter ego, sympathy, intersubjectivity, praxis
\end{abstract}

A continuación se hace un estudio del así denominado problema de las otras mentes (POM) desde una perspectiva humeana, a través de la exégesis y reelaboración crítica de algunas de las tesis defendidas por David Hume en el marco de su filosofía. ${ }^{1}$ Se busca extraer princi-

${ }^{1}$ Por cuestiones de conveniencia, se restringirá este abordaje a algunos de los pasajes más relevantes de $A$ Treatise of Human Nature. Para hacer referencia a 
palmente algunos corolarios, teóricos y metateóricos, relativos al anticartesianismo que se evidencian por las indicaciones y la impronta de Hume. Para ello, se divide el trabajo de la siguiente forma. En la sección II se caracterizará el POM un poco más detalladamente, buscando reconstruir en unas cuantas líneas su origen cartesiano y las principales problemáticas que hereda de dicho origen.

En la sección III se reconstruirá la opinión de Anthony Pitson, uno de los pocos intérpretes de Hume que ha puesto especial atención en el problema de las otras mentes desde una perspectiva humeana. Con ello se intenta recuperar la importancia que en dicha explicación adquiere el mecanismo de la simpatía. Inmediatamente después, en la sección IV se darán algunas razones generales por las cuales dicha noción resulta clave para comprender el giro anticartesiano que se deriva de algunas de sus propuestas más relevantes, y se planteará y responderá una objeción a dicha lectura.

Posteriormente, en la sección V se resaltará la importancia que adquieren, desde la perspectiva humeana, el ámbito intersubjetivo y la dimensión pasional de la subjetividad. Ambas características permitirán perfilar uno de los movimientos metateóricos decisivos de la discusión humeana en torno a la subjetividad: una especie de giro práctico que parece imponerse, en oposición a la forma tradicional en que el cartesianismo abordaba la relación teoría-praxis. Por ende, en esta instancia se revalorizará la propuesta humeana como una alternativa metateórica al cartesianismo, lo cual permitirá rescatar su pertinencia y actualidad en relación con algunas de las aproximaciones críticas anticartesianas del siglo pasado.

Por último, en la sección VI se mostrará cuán novedosa, compleja e interesante resulta (esta lectura de) la teoría humeana de la subjetividad y de la intersubjetividad, especialmente en el marco de las discusiones contemporáneas que, por lo general, no toman en consideración posibles aportes desde perspectivas humeanas.

esta obra se la llamará Tratado, y en las referencias bibliográficas se abreviará con una T seguida del número de libro, la parte, la sección y el párrafo, y se incluirá el número de página de la edición clásica de Selby-Bigge. De esa forma, T1.1.1.1, SB 1 ha de leerse Tratado de la naturaleza humana, libro 1, parte 1, sección 1, párrafo 1, página 1 de la edición Selby-Bigge. En todos los casos, las traducciones de las citas del Tratado son mías.

Diánoia, vol. LIX, no. 72 (mayo de 2014). 
Lo que aquí se denomina POM es un problema filosófico relativo a la existencia de otros sujetos u otros "yo" (además de uno mismo), legado a la posteridad de la historia de la filosofía a causa de algunos complejos vericuetos argumentativos e implicancias teóricas de la filosofía de René Descartes. ${ }^{2}$ Como se verá a continuación, dicha herencia cartesiana haría del POM, al menos en lo fundamental, una dificultad epistemológica. ${ }^{3}$

El POM es el problema de la constatación de la alteridad; pero no de cualquier alteridad, sino de la alteridad de otro yo humano. Descartes parece no encontrar dificultades teóricas en fundar el carácter necesario y absoluto de la Alteridad (con mayúscula), pues ha podido demostrar sin lugar a dudas la existencia necesaria de Dios; ${ }^{4}$ pero no sucede lo mismo con la alteridad entendida en términos de otros sujetos finitos humanos: en otras palabras, el problema de las otras mentes es el problema de la humanidad del otro que aparece ante mí.

Dicha dificultad epistemológica, según suele sostenerse, proviene del carácter representacionista de la filosofía cartesiana y tradicionalmente ha sido un inconveniente que arrastran todas las teorías que comparten esa misma característica. Se puede enunciar concisamente de la siguiente manera: el fenómeno del reconocimiento (que el sujeto reconozca a otro como par, es decir, como otro sujeto en pie de igualdad) está subordinado a la representación del otro que cada sujeto es capaz de hacer. Toda aprehensión que puedo tener del otro está siempre mediada o atravesada por la representación; por lo tanto, el otro nunca aparece primaria e inmediatamente como lo que efectivamente es, i.e., un sujeto, sino que siempre es primero un objeto de mi representación, por lo cual se abre siempre un abismo entre el ego cogitans (o sujeto en primera persona) y el alter ego, cuyo estatus nunca se manifiesta ante el ego cogitans en su plenitud, sino que pareciera que constantemente tiene que ser inferido. Por lo tanto, al tratarse siempre de un objeto de mi juicio o de mi razonamiento, la opinión que pueda formar de aquel

${ }^{2}$ Aunque en la bibliografía se consignan las más conocidas traducciones de la obra de Descartes al castellano, se citará a través de la edición canónica de Adam y Tannery de las Obras completas, con la abreviatura AT, seguida del número de tomo y de la página; por ejemplo: AT, IX, 51 (tomo IX de la edición Adam/Tannery, página 51).

${ }^{3}$ Cfr. Pitson 1996, y Waldow 2009, pp. 4 y ss.

${ }^{4}$ Cfr. AT, VI, 33-35; AT, IX, 27-38 y 38-42; AT, IX, 52-53.

Diánoia, vol. LIX, no. 72 (mayo de 2014). 
objeto que tengo frente a mí (a saber, que se trata de otro sujeto) nunca está exenta de error. ${ }^{5}$

Aunque lo dicho es poco, ya es posible advertir sin mayores obstáculos cómo, a partir de esta caracterización, se perfila una serie de cuestionamientos de índole epistemológica que han resultado considerablemente incisivos para el cartesianismo en particular, pero que (como se ha sugerido) han logrado trascender el ámbito estricto del cartesianismo y se han perpetuado como problemas teóricos en perspectivas que comparten con aquél la impronta representacionista e individualista. Entre otros, se pueden mencionar los siguientes: (a) ¿qué razones (o evidencias) tenemos para concluir que existen, de hecho, otros sujetos además de mí?; (b) ¿cuál (o cuáles) entre nuestras facultades es(son) la(s) que nos garantiza(n) un conocimiento del otro en cuanto alter ego?; (c) ¿qué tipo de conocimiento tenemos del otro en cuanto alter ego?

Del mismo modo, puede entreverse también una cuestión que adquirirá mayor relevancia en el transcurso de este trabajo. Me refiero a algunas de las estrategias metateóricas que han adoptado filósofos contemporáneos (especialmente del siglo pasado) para analizar y criticar el tipo de abordaje propugnado por el cartesianismo (heredado en gran medida por toda una tradición moderna). Desde esta aproximación epistemológica e individualista, en la que el reconocimiento del otro queda supeditado a mi representación del otro, se efectúa una suerte de reducción del otro a mí mismo, a las categorías que le imponen mi representación estructurante. Toda diferencia parece tener que ser resuelta en cierta regla de identidad o mismidad que es propiedad o prerrogativa del sujeto en primera persona (del ego cogitans) y que se aplica a "objetos externos" por igual (sean éstos objetos materiales u "objetos-sujetos"). Aunque resulta imposible profundizar en esta dimensión metateórica como correspondería en esta oportunidad, sí se tendrá presente en lo que se dirá a continuación. Me refiero, por ejemplo, a ciertas tesis como las de E. Lévinas, quien bregaba por una aproximación fundacional y fundamentalmente ética al Otro como la vía que posibilitaría romper la asimetría que jerarquiza a la primera persona (desde la perspectiva epistemológica moderna). ${ }^{6}$

${ }^{5}$ Como sí lo está la constatación intuitiva e indubitable de mi propia existencia en cuanto ego cogitans. Cfr. AT, VI, 32-33; AT, IX, 19-22.

${ }^{6}$ Cfr. Morgan 2011, pp. 114 y ss.; Pérez Quintana 2008, y Peperzak 1993, pp. 49 y ss. Cabe aclarar que se menciona a Lévinas y su filosofía únicamente a modo ilustrativo, es decir, como ejemplo conspicuo de lo que aquí se denomina una inversión en el plano metateórico. Por lo tanto, es indispensable tener en cuenta que (a) éste

Diánoia, vol. LIX, no. 72 (mayo de 2014). 


\section{III}

Al buscar apoyo textual concreto sobre la opinión de Hume respecto del POM, dos cuestiones saltan inmediatamente a la vista. En primer lugar, siguiendo un poco por analogía la estrategia de Hume a lo largo de T1, se esperaría que mostrara la irrelevancia de las demostraciones filosóficas de la existencia de otros sujetos independientes a nosotros para luego explicar, no si de hecho existen otros sujetos, sino, en cambio, cómo es que llegamos a formarnos la creencia en su existencia. ${ }^{7}$ Pues bien, eso nunca sucede, ni en el Tratado, ni en ninguna otra obra de Hume. Nunca se refiere directa o principalmente al tema, salvo algún comentario subsidiario al tratar temas afines, como el de las pasiones ${ }^{8}$ o el de las virtudes artificiales. ${ }^{9}$

En segundo lugar, debido justamente a esto último, es prácticamente innegable que Hume da por sentada la posibilidad de dicha creencia a lo largo de todo el Tratado (más aún, a lo largo de todos sus escritos filosóficos): el hecho mismo de que tengamos una creencia en la existencia de otras mentes pareciera innegable para Hume, puesto que muchos de los mecanismos mentales que se esfuerza por explicar únicamente funcionan sobre la base (implícita) de dicha creencia. ${ }^{10}$

Aunque esto parece evidente, las consecuencias que pueden extraerse (y se han extraído) al respecto no siempre son coincidentes. Puede pensarse que la razón por la cual Hume no hace ninguna mención del tema es porque carece de una explicación satisfactoria (Penelhum 2000 , p. 52); o bien porque se trata de una explicación que puede reducirse a otra de mayor generalidad, como nuestra creencia en los objetos externos (eso parece ser lo que piensa Kemp Smith al respecto, cfr. 1941, p. 124), o bien porque, una vez abandonados los temas metafísicos del libro 1 y adentrados ya en las temáticas de corte social e

no es un trabajo dedicado a Lévinas ni a alguna de sus propuestas filosóficas, y (b) no se intenta trazar ningún tipo de ecuación, explícita o implícita, entre el filósofo franco-lituano y David Hume.

${ }^{7}$ Con ello suponemos implícitamente que Hume podría llevar a cabo un argumento similar al que propone para argumentar, no la existencia de los cuerpos, sino nuestra creencia en ellos. Cfr. T1.4.2.

${ }^{8}$ Especialmente las pasiones indirectas que tienen por objeto a otros sujetos (amor/odio), cfr. T2.1.2.

${ }^{9}$ Véase particularmente T3.2.2, donde Hume discute ciertas prácticas y conductas coordinadas de forma intersubjetiva que dan origen a nuestra idea de justicia.

${ }^{10}$ Entre otros, por ejemplo, nuestra idea de propiedad privada (T2.1.9 y T3.2.2), pero especialmente el mecanismo de la simpatía, del cual se hablará con un poco más de detalle a continuación. 
interpersonal de los libros 2 y 3 , el problema se disuelve y nunca reaparece en el curso ordinario de nuestras vidas (Baier 1991, pp. 138-140).

Muy pocos intérpretes han considerado tomar otro camino: cuestionarse por la posibilidad de desarrollar una explicación de dicha creencia sobre la base de los principios de la filosofía de Hume, de forma tal que se ofrezca a la vez una interpretación/explicación consistente con ella.

La propuesta de A.E. Pitson (1996, y 2002, cap. 8) fue una de las primeras, y continúa siendo uno de los pocos intentos de formular una explicación de esta creencia fundamental dentro de esquemas humeanos. Para garantizar la adecuación con lo efectivamente dicho por Hume, Pitson propone adoptar la estructura general de T1.4.2 (la sección dedicada a nuestra creencia en objetos externos) e indagar hasta qué punto es viable hacerla funcionar para esta otra explicación. En otras palabras, copiar el mismo esquema de la explicación humeana de nuestra creencia en la existencia continua y distinta de los objetos externos y ver cómo puede adaptarse para explicar la creencia, por así decir, en la existencia continua y distinta de otras mentes.

La ventaja de sus sugerencias sobre el tema es que, en todo momento, Pitson es muy cuidadoso y medido en su exposición, evitando compromisos teóricos fuertes con algunas de las vertientes más recurrentes dentro de la hermenéutica de los textos humeanos. El resultado es un conjunto bastante sólido y que, muy brevemente, aporta mucha luz al tema del POM en una perspectiva netamente humeana, al punto de que otra de las pocas publicaciones dedicadas al tema, la de A. Waldow (2009), reconoce su deuda con el texto inaugural de Pitson. También reconoce la importancia de la simpatía en una explicación que dé cuenta cabalmente de la creencia en otras mentes como una creencia natural. Sin embargo, a diferencia de la estrategia de Pitson, que implica pensar la simpatía como un mecanismo imaginativo y, por ende, oponerlo a cualquier tipo de razonamiento o inferencia, Waldow sostiene que la crítica humeana a la metafísica impediría una distinción tan tajante entre imaginación y razón, lo cual lo lleva a aproximar el mecanismo de la simpatía a la facultad responsable de nuestras inferencias, que en varias oportunidades Hume denominaría indistintamente o bien razón o bien imaginación (cfr. Waldow 2009, pp. 4, 48 y ss., 73 y ss., 140 y ss.).

No obstante, la propuesta de Waldow se acerca de forma mucho más marcada al conjunto de interpretaciones que suelen denominarse "realistas escépticas" (cfr. Wright 1983, o Strawson 1989 y 2011). Desde este tipo de interpretaciones, la existencia de las entidades a las que Hume hace referencia dentro de sus argumentos escépticos (en este 
caso, las otras mentes) no sería nunca de hecho puesta en duda por él, sino únicamente el tipo de conocimiento que tenemos de ellas; para expresarlo mejor, aquello que es objeto de sus ataques escépticos es la idoneidad o aptitud de nuestras facultades mentales para poder darnos un conocimiento que, por lo general, no están capacitadas para darnos.

Esta vertiente interpretativa es muy discutida en la actualidad, tanto en contra como en su defensa; pero en esta oportunidad no nos abocaremos a ella, se dejará de lado la propuesta de Waldow y únicamente se hará referencia muy brevemente a la de Pitson, con la intención de establecer algunas cuestiones fundamentales respecto del POM en clave humeana, con base en la cual se discutirán las implicaciones filosóficas ulteriores a la luz de lo que se dijo en la sección anterior.

En primer lugar, la primera línea de continuidad que traza Pitson corresponde al contenido que han de presentar nuestras percepciones. En el caso de los objetos externos, estamos al tanto de su estrecho vínculo con el problema de la identidad, es decir, para que nuestra mente se vea inclinada a considerar un objeto como el mismo a través del tiempo, éste tiene que mostrarse continua e ininterrumpidamente. Lo mismo, entonces, tendríamos que considerar en el caso de las otras mentes: el carácter invariable e ininterrumpido es una parte vital de la noción de identidad (en general) y, por ende, sería también un componente insoslayable de nuestra creencia en otras mentes, mentes que existen a través del tiempo, continuas e independientes de nuestra percepción. ${ }^{11}$

Una vez estipulado esto, lo siguiente es indagar cuál entre nuestras facultades es la responsable de dicha creencia. Del mismo modo que Hume en T1.4.2, Pitson recorre tres alternativas: los sentidos, la razón y la imaginación. La intención es, tal como hace Hume en el caso de los

${ }^{11} \mathrm{Al}$ referirnos a mentes, este carácter ininterrumpido e invariable no parece ser tan evidente como en el caso de los cuerpos externos. Esta diferencia podría indicar, aunque no sea más que vagamente, la complejidad que conlleva un cambio de registro tan importante como el que supone hacer funcionar un marco teórico originalmente pensado para dar cuenta de la existencia continua y distinta de objetos externos (que se presentan con determinadas características, especialmente las de ser visibles y tangibles) para explicar la existencia continua y distinta de otros sujetos (es decir, individuos que no sólo son otros cuerpos "similares" al nuestro, sino que son además otras mentes "similares" a la nuestra, siendo estas últimas invisibles e intangibles). Aunque esta situación sin duda genera una tensión (teórica y explicativa) desde el arranque, nos presenta de forma inequívoca el vínculo que hemos de analizar y que será precisamente un punto neurálgico hacia el cual estará dirigida la discusión sobre la simpatía, i.e., el vínculo que puede trazarse entre cuerpos y ("sus") mentes. 
objetos externos, descartar los sentidos y la razón para argumentar en favor de un origen en la imaginación.

Pitson da dos argumentos contundentes para desestimar los sentidos. En primer lugar, siguiendo a Hume, la intención es mostrar cómo se forma en nuestra mente la idea de algo (objetos externos o mentes) que continúa de forma ininterrumpida en la existencia cuando no lo estemos percibiendo. Pero sostener que los sentidos nos dan una idea de existencia de algo que, ex hypotesi, no es percibido por nuestros sentidos resulta un tanto paradójico.

Además, hay otra consideración de peso al respecto, relacionada más cercanamente con el POM. Si la idea que estamos buscando efectivamente nos fuera transmitida a través de los sentidos, sucedería entonces que podríamos tener una percepción directa de otras mentes, lo cual no sólo rechaza Hume en más de una oportunidad (por ejemplo, T2.2.1.1, SB 329, y T3.2.1.2, SB 477) sino que, asimismo, eliminaría de raíz el "problema" del POM, ya que, como se describió en la sección II, el POM radica precisamente en que únicamente podemos percibir de forma directa cuerpos y sus comportamientos, es decir, conductas y disposiciones corporales, pero nunca "otras mentes".

Descartar la razón como facultad causante de la idea que buscamos ya supone un esfuerzo más cuidadoso y que requiere mayor detenimiento. Pitson está interesado en excluir cualquier forma de razonamiento como causante de esta idea, pero siendo ésta una cuestión de hecho, le preocupa especialmente mostrar que no llegamos a dicha idea a través de ninguna analogía o razonamiento analógico. La forma en que sostiene esta tesis es, a mi entender, muy interesante. Todo razonamiento está compuesto, según Hume, de una comparación (entre ideas, o entre ideas y una impresión presente en la memoria o los sentidos). ${ }^{12}$ El otro componente importante de todo razonamiento es la semejanza. Para poner en relación o comparar una o más percepciones, la mente ha de encontrar cierto grado de semejanza o cierto elemento coincidente entre ellas. ${ }^{13} \mathrm{El}$ razonamiento analógico es, en este sentido, una comparación entre dos o más ideas con base en una semejanza que no es total o general (sino, por ejemplo, gradual o limitada). ${ }^{14}$ Por ende, la conclusión se infiere, o se deriva con cierto grado de probabilidad, o con cierta diferencia producto de la pequeña divergencia entre los elementos de la comparación.

${ }^{12}$ Véase T1.3.2.2, SB 73-74.

${ }^{13} \mathrm{~T} 1.3 .2 .2$, SB 73-74.

${ }^{14}$ Véanse especialmente T1.3.12.25, SB 142, y T1.3.13.9, SB 148.

Diánoia, vol. LIX, no. 72 (mayo de 2014). 
Pero, entonces, para poder llevar a cabo una analogía tendríamos que disponer ya de las ideas que vamos a comparar, en este caso, de una mente. Pitson hace notar que Hume sí utilizaría explícitamente el razonamiento analógico para inferir la posesión de una mente para el caso de los animales, ${ }^{15}$ pero no así con los seres humanos (nunca se hace referencia a ningún razonamiento). Pitson entiende que ello significaría que, para derivar la existencia de mentalidad en los animales, ya habríamos de detentar una idea de "otras mentes (humanas)", y sería sobre este conjunto previo de ideas que inferiríamos (por analogía) la existencia de otras mentes para el caso de los animales. La analogía misma es posible, sin duda, porque hay una gran similitud entre los animales y nosotros los hombres, pero también (y quizá más importante aún para un razonamiento analógico), porque no hay una similitud total, sino que hay aspectos en los que diferimos.

Para explicar la idea de otras mentes (humanas) que hemos obtenido en un primer momento, necesitamos apelar a otro mecanismo: la imaginación. En este momento entra en juego el mecanismo de mayor importancia para Pitson (y en eso estoy de acuerdo con él) en la formación de la creencia que estamos indagando: la simpatía. Este mecanismo no es otra cosa que la capacidad de recibir por comunicación los sentimientos y disposiciones del otro, de forma tal que, a través de un esfuerzo de la imaginación, podemos insuflar de vivacidad una idea al punto de transformarla en una impresión. ${ }^{16}$

La explicación que Pitson considera más adecuada, y que se ajustaría mejor a los propios estándares explicativos humeanos tal como podemos elucidarlos en T1.4.2, es que la creencia en la existencia continua y distinta de otras mentes es un producto de la imaginación y, especialmente, de la simpatía. Para resumirlo concisamente, podríamos expresarlo del siguiente modo:

1) Tenemos, en la experiencia interna, ideas tanto de nuestras conductas (acciones corporales) como de nuestras operaciones o estados mentales, todas derivadas de sus impresiones previas correspondientes. Pero también tenemos experiencia de múltiples conjunciones constantes entre algunas de las primeras y algunas de las segun-

${ }^{15}$ Quizá el pasaje más claro al respecto sea T1.3.16.2, SB 176.

${ }^{16}$ Hay muchos estudios sobre la simpatía, especialmente centrados en el desarrollo de esta noción durante el siglo XVIII, orientados a diversos aspectos (filosóficos, psicológicos, sociales, históricos). Como ejemplos, véanse Frazer 2010; Lamb 2009, y Gottlieb 2007. También hay un interesante capítulo dedicado a la simpatía humeana (donde también se la pone en relación con la noción smitheana de simpatía) en Árdal 1966, cap. 4. 
das, es decir, conjunciones constantes de estados mentales seguidos de acciones corporales, o viceversa.

2) Tenemos también ideas que corresponden a cuerpos externos (i.e., creemos en su existencia independiente de nuestra percepción) extremadamente similares a los nuestros y que asimismo llevan a cabo acciones muy semejantes a las nuestras (ideas, éstas también, que provienen de sus correspondientes impresiones). Ahora bien, estas ideas de acciones corporales, por su semejanza con las nuestras, nos llevan a considerar las ideas que tenemos de sus acompañantes habituales, es decir, las ideas de las operaciones mentales que por lo general consideramos sus causas o sus efectos.

3) Es aquí donde entra en juego la simpatía. El vínculo entre nuestras ideas de conductas y nuestras ideas de eventos mentales no es un razonamiento analógico, no hay una comparación y una inferencia gradual, sino que se trata de una relación causal, es decir, un mecanismo de asociación. Pero, como no hemos tenido nunca (ni podremos tener, por principio) ninguna impresión de "otra mente", la creencia es producto de la simpatía, es decir, de un esfuerzo de nuestra imaginación que le transmite vivacidad a partir de la "idea vivaz" (o impresión) siempre íntimamente presente que tenemos de nosotros mismos (en cuanto sistema de percepciones fugaces), haciendo de la idea que nos formamos de los sentimientos de los demás una impresión fuerte y vivaz (o, en otras palabras, una creencia en la realidad de los estados mentales de los otros sujetos).

Este breve panorama nos proporciona una noción general de cómo puede concebirse una respuesta factible al POM dentro de un marco que, más allá de los inconvenientes que pudiera llegar a presentar, es sin duda humeano en su espíritu. Es suficiente, en todo caso, porque sobre la base de estas indicaciones podemos hacernos las preguntas que originariamente nos interesaba hacer, entre ellas si la incorporación de un mecanismo de la imaginación (como es la simpatía) resulta viable para responder al problema cartesiano de las otras mentes.

\section{IV}

Si gran parte del POM, como aquí se ha argumentado, surge a partir de la perspectiva internista y basada en la primera persona (el otro parece no poder dejar de ser una representación mía y nunca podemos tener acceso inmediato al otro como sí sucede con el acceso intuitivo

Diánoia, vol. LIX, no. 72 (mayo de 2014). 
que tenemos respecto de nuestra propia existencia), entonces, en principio pareciera que al eliminar de raíz cualquier noción de intuición intelectual y al incorporar la imaginación y la simpatía en su explicación (mecanismos que nos permiten experimentar o vivenciar, y no ya concebir o representarnos las sensaciones de los otros), ${ }^{17}$ la propuesta de Hume tendría algunas claras ventajas respecto de la cartesiana en relación con el POM. Sin embargo, parece posible reconstruir, en una serie de pasos, una elaborada objeción contra esta lectura.

1) Para funcionar, el mecanismo de la simpatía requiere disponer, previamente, de dos elementos: a) la idea a la que ha de insuflarse fuerza y vivacidad (para transformarse en impresión), y b) una fuente de vivacidad que haga posible la transmisión.

2) El primer elemento nos es provisto por la experiencia que tenemos de las conductas y acciones de cuerpos externos. El segundo elemento lo incorporamos nosotros, debido a la impresión interna y constante que tenemos de nosotros mismos. Únicamente a partir de ella podemos avivar la idea de sentimientos o pasiones de otra mente al punto de llegar a una creencia en que, detrás de las acciones y conductas de aquellos cuerpos, hay mecanismos y operaciones mentales que funcionan ora como causas, ora como efectos.

3) Pero, entonces, Hume tampoco puede desembarazarse de esa primacía de la primera persona: nuestros propios contenidos mentales, nuestras propias emociones y sentimientos son requisitos ineludibles para formarnos una idea (vivaz) de las emociones y sentimientos de los demás. Más aún, sin las primeras no podríamos nunca formar una creencia en las segundas. El problema de la diferencia

\footnotetext{
${ }^{17}$ Algunos intérpretes han sostenido que las impresiones, al ser percepciones que surgen originariamente en la mente y que no proceden de ninguna otra percepción, ni son copia ni representan alguna, no tendrían un carácter representativo, sino que serían únicamente un motor de la mente que pone en movimiento una serie de mecanismos que producen, de forma secundaria, ideas, es decir, contenido representativo. Por ende, al hacer hincapié en la importancia de las impresiones como aquello que pone en movimiento la mente, como causa de las tendencias, inclinaciones y propensiones de la mente, Hume estaría bosquejando (implícita o explícitamente) una crítica a las teorías (como la cartesiana y la lockeana) que defienden la preeminencia absoluta de los fenómenos representativos y del carácter representativo de los contenidos mentales. Cfr., entre otros, Deleuze 1977, pp. 1129, especialmente la p. 22, y Martínez de Pisón 1992, cap. 2.
} 
entre la primera y el resto de las personas, por ende, persistiría y parecería que nuevamente el conocimiento que tenemos de los demás sería únicamente posible si está atravesado de un extremo al otro por el conocimiento que tenemos, primeramente, de nosotros mismos.

Hasta aquí la objeción. Los puntos (1) y (2) han sido confirmados por el comentario de la reconstrucción que Pitson hace del tema y no hay que renegar de ellos. Para rebatir esta objeción, por ende, hemos de proponer una alternativa que desarticule la aparente implicación que habría entre ambos y el punto (3), que sí será el punto clave de la discusión. Para ello, será conveniente establecer algunas premisas correspondientes a la teoría humeana de la subjetividad, lo que nos obliga a hacer una pequeña digresión.

Comencemos estableciendo que Hume rechaza cualquier forma de intuición intelectual, incluyendo una que capte al propio sujeto ( $\mathrm{T}$ Int. 8, SB xvii; T1.4.6.1-4, SB 251-253). No existe ningún acceso privilegiado, inteligible o racional, al entendimiento ni objetos acordes a dicho acceso (al menos, hasta donde nos es posible conocer a nosotros los hombres). Los únicos contenidos de nuestra mente son percepciones: impresiones e ideas (primarias y secundarias, de los sentidos, de la memoria o de la imaginación). Estas percepciones, sumadas a los principios de asociación, son los únicos elementos que componen nuestro mundo y el "cemento" que los une. ${ }^{18}$

Esto último implica que únicamente percibimos percepciones (valga la redundancia), pero en ningún caso percibimos "mentes". Podemos formarnos una idea de mente como colección o sistema de percepciones unidas por lazos de causalidad (T1.4.6.19); pero, más allá de esta "impresión de nosotros mismos" como sucesión de pensamientos, sentimientos y acciones, nos es imposible aprehender la naturaleza última de nuestra mente. Todas nuestras percepciones forman parte del haz o corriente de percepciones que integran el sistema; por ende, entran en relación causal unas con otras y serán siempre consideradas, en un aspecto al menos, "nuestras" y nunca de alguien más. Aunque perciba un cuerpo externo a mí, igualmente considero que esa percepción en sí misma es "mía" y de nadie más (en el sentido en que forma parte del haz que conforma mi mente). Por lo tanto, no podemos percibir más

${ }^{18}$ T1.1.1.1, SB 1. Respecto del término "cemento del universo", se trata de una expresión que Hume utiliza en el Resumen del tratado de la naturaleza humana para referirse a los principios de asociación; cfr. la edición de Norton y Norton (Hume 2000), p. 417.

Diánoia, vol. LIX, no. 72 (mayo de 2014). 
que los elementos componentes de "nuestro" haz o sistema de percepciones (nuestra mente) y nunca directamente percepciones externas o exteriores al haz.

Pero, entonces, no tenemos (en sentido estricto) ningún acceso privilegiado o conocimiento certero y simple de (la naturaleza de) nuestra mente. Muy por el contrario, se trata simplemente de una idea que formamos a partir de un conjunto más bien complejo de elementos, los cuales únicamente nos son dados a conocer a través de sus operaciones en la experiencia y, en muchos casos, gracias a conjunciones constantes y contracción de hábitos. Podría decirse que nuestras percepciones son privativas de nuestra mente y sólo nosotros tenemos acceso a ellas (es decir, sólo nosotros las percibimos, pues si las percibiera otro, no serían nuestras) y, por ende, en este sentido tenemos un acceso privilegiado a ellas pero no de la forma en que creía Descartes (intuición intelectual inmediata e indubitable de nuestra existencia y de la naturaleza sustancial simple e inmaterial de la misma).

Por otro lado, hacer hincapié en el fenómeno de la simpatía nos abre a toda una nueva dimensión de la teoría de la subjetividad de Hume que ha sido, en gran medida, parcial o totalmente desatendida por la gran mayoría de los más renombrados intérpretes de Hume. Si es cierto que estos últimos restringen sus análisis de la teoría humeana del sujeto a T1 y al apéndice de T3, ofreciendo como corolario una lectura extremada y exclusivamente mentalista del tema, ${ }^{19}$ no es menos cierto que, al incorporar la teoría humeana de las pasiones tal como es presentada en T2, hemos de examinar y dar cuenta de una serie de fenómenos subjetivos de carácter pasional que provocan un novedoso reajuste en la tradicional caracterización del sujeto humeano. Éste parece no ajustarse más a la lectura distorsionada que hace de él un haz fugaz y perecedero, desprovisto de unidad y continuidad, sino que se muestra como una unidad agente, provista de un cuerpo, de emociones, sensaciones y pasiones que inclinan su voluntad y funcionan como condición de posibilidad de gran parte de sus facultades y prácticas. Dentro de este nuevo campo, la simpatía desempeña un papel de im-

${ }^{19}$ Entre estos enfoques restrictivos que desestiman cualquier papel para la dimensión pasional de la subjetividad en el planteo humeano se encuentran los de Stroud (1977), Fogelin (1985), Garrett (1997), Noonan (1999, 2007) y Strawson (2011). Véanse T1.4.6.5 y T1.4.5.19, los pasajes donde Hume hace referencia a la "identidad personal con respecto a nuestras pasiones o el interés que tenemos por nosotros mismos", en contraste con la "identidad personal con respecto a nuestro pensamiento o imaginación". 
portancia, pues es en gran medida a través de ella que las pasiones nos son comunicadas. ${ }^{20}$

Ninguna cualidad de la naturaleza humana es más notable, tanto en sí misma como por sus consecuencias, que la propensión que tenemos a simpatizar con otros y a recibir por comunicación sus inclinaciones y sentimientos, sin importar cuán distintos o incluso opuestos a los nuestros sean. [...] Odio, resentimiento, estima, amor, coraje, júbilo y melancolía, todas las pasiones las siento más por comunicación que por mi propio temperamento y disposición naturales. (T2.1.11.2, SB 316-317)

Esta apertura al papel insoslayable de la dimensión pasional dentro de los procesos graduales que dan forma a nuestra subjetividad implica un reconocimiento y una revaloración tanto de los otros sujetos como del mecanismo mismo de simpatía, a través del cual podemos experimentar sus emociones. Ambos se tornan indispensables: la intersubjetividad y el principal mecanismo de interacción que la posibilita (la simpatía) son ambas fundamentales en la formación de nuestra subjetividad. Ello es así porque las pasiones mismas, la vida emocional del hombre, únicamente son posibles en relación con otros. El sujeto únicamente surge como tal dentro de un contexto pasional, es decir, intersubjetivo, un contexto social previo que se muestra mayor que él (ya existía antes que él y, presumiblemente, continuará existiendo cuando ya no esté).

Quienes disfrutan al pronunciarse en contra de la naturaleza humana han observado que el hombre es con todo insuficiente para sostenerse a sí mismo y que cuando se lo separa de todo vínculo con objetos externos, inmediatamente se hunde en la más profunda melancolía y desesperación. [...] Hasta tal punto estoy de acuerdo que considero que la mente es insuficiente, en sí misma, para su propio entretenimiento y busca naturalmente objetos externos. [...] Es por eso que la compañía es naturalmente tan agradable, pues nos presenta el más vívido de todos los objetos, a saber, un ser racional y pensante como nosotros mismos, que nos comunica todas las acciones de su mente, nos pone al tanto de sus más íntimos sentimientos y afecciones y nos deja apreciar, en el mismo instante de su producción, todas las emociones causadas por cualquier objeto. (T2.2.4.4, SB 352-353)

${ }^{20}$ Algunos estudios sí han reparado en esta otra dimensión de la subjetividad; pueden encontrarse algunas sugerencias en Baier 1991, en la lectura de McIntyre (2009) y en algunas propuestas del mismo Pitson (2002). El carácter social del sujeto humeano fue defendido por J. Baillie (2000, pp. 31 y ss.).

Diánoia, vol. LIX, no. 72 (mayo de 2014). 
Sin estos otros sujetos, las pasiones languidecerían y morirían (T2.2.5. 15, SB 363 es rotundo al respecto) y, junto con ellas, también lo haríamos nosotros. La dimensión de mayor peso en nuestra vida psíquica, la pasional, es enteramente dependiente en su origen, desarrollo, continuidad y complejización de este otro ámbito, de raigambre social, compuesto por otros sujetos con los cuales (inter)actuamos, hacia los cuales se refieren casi necesariamente nuestras acciones y conductas y en relación con los cuales (debido a que simpatizamos con ellos) tomamos decisiones, modificamos costumbres o corregimos puntos de vista (cfr. T2.2.1-2, T3.2.2, T3.3.1).

La dinámica social que presenta Hume a partir de T2 y que a medida que avanza el Tratado va sumando capa tras capa de elaboradas caracterizaciones se transforma en el contexto desde el cual surge la subjetividad individual. El sujeto es sujeto sólo dentro de una comunidad interrelacionada de sujetos que se vinculan con él en diversos niveles (mental, pasional, moral, político, estético, científico e incluso histórico).

La subjetividad y la individualidad son un producto de relaciones intersubjetivas que se dan dentro de una comunidad de sujetos vinculados y vinculantes los unos con los otros. Como corolario, dependerá mucho en qué comunidad vivamos para saber qué sujetos somos. En otras palabras, no se es sujeto desde siempre, sino que se llega a ser sujeto; y tendremos que saber en qué sociedad hemos nacido y nos hemos formado para poder decir algo significativo y privativo sobre nosotros mismos. Aunque no podamos detenernos a glosar en detalle la rica argumentación humeana, cabe mencionar que, además del fenómeno de la comunicación simpática, hay algunas otras cuestiones que Hume analiza y que aportan gran apoyo a estas consideraciones, entre ellas: la relevancia de la propiedad privada dentro del mecanismo que produce las pasiones del orgullo y la humildad (cfr. T2.1.10.1, SB 309-310), y la importancia máxima que adquieren la opinión y los sentimientos de los demás cuando las pasiones influyen sobre nosotros ( $c f r$. T2.1.6, SB 290-295).

A través de los juicios que se forman sobre nosotros los sujetos con quienes nos relacionamos es como vamos elaborando nuestra idea de la propia reputación, carácter o nombre. Estos términos, muy asociados, dan una clara muestra de cómo es que nos consideramos más felices o afortunados si así lo juzgan los demás.

Aun con grandes ventajas, la soledad hace del hombre un ser infeliz: la soledad, el solipsismo o el sufrir el rechazo del círculo social 
hacen del hombre un sujeto "malformado", ${ }^{21}$ un "monstruo" tal como el propio Hume lo presenta en T1.4.7. Los placeres que el filósofo pudo obtener en su actividad filosófica se muestran poco duraderos si se ponen al margen de la sociabilidad. Al haber perdido el mundo, el filósofo languidece y entra en desesperación. Nuestra subjetividad sólo puede recibir un contenido específico dentro del mundo, i.e., en un grupo social particular, y a través de un marco de reglas, normas, estándares y reciprocidad entre individuos. Aisladas del grupo social que nos contiene y dentro del cual surgieron, nuestras reglas generales, normas jurídicas y estándares (de todo tipo: epistémicos, morales, estéticos) pierden todo su sentido y propósito.

De esta forma, Hume lleva a cabo un desplazamiento clave: el abandono de la prioridad de la primera persona. Ya en su vertiente cartesiana, ya en su vertiente lockeana, Hume ve con desconfianza la excesiva preponderancia otorgada a la perspectiva de la primera persona (individualista, privilegiada, primordial, reflexiva, racional, privada, voluntarista, absoluta). Estos tres fenómenos, entre otras cosas, i.e., la simpatía, la apertura hacia los sentimientos y las opiniones de los otros y la forma en que dicha opinión repercute en la formación de nuestra personalidad, permiten a Hume dotar de consistencia suficiente a una alternativa que haga de la subjetividad un resultado más que un presupuesto, y el logro conjunto de varios y complejos factores que por lo general son enteramente contrarios a los que recién hemos enlistado: no individualistas (sino intersubjetivos o sociales), no reflexivos (prerreflexivos e impersonales), no racionales (pasionales y emotivos), no privados (el carácter privado se construye sobre el fondo de lo públicamente observable y sus efectos), antivoluntaristas (involuntarios, mecánicos, instintivos). El sujeto nunca puede ser concebido despojado por completo de toda relación, sino más bien a la inversa: para ser pensado como sujeto, debe pensarse sujeto a una compleja trama de relaciones intersubjetivas.

Esta alternativa humeana aquí delineada, pues, se erige simultáneamente en contra del cartesianismo, respondiendo a la posible objeción que se había adelantado al inicio de este apartado: no podemos tener un conocimiento del alter ego que resulte inmediato, incondicional y totalmente desvinculado de cualquier relación extrínseca, pero porque un conocimiento tal no existe, su postulación es una falacia, una abstracción vacía de cuño racionalista que no puede cumplirse ni siquiera

${ }^{21}$ En el sentido de "deforme", aunque también cabe el juego de palabras respecto a la malformación de la subjetividad.

Diánoia, vol. LIX, no. 72 (mayo de 2014). 
con nosotros mismos. En otras palabras, no tenemos un conocimiento inmediato y puro (en sentido cartesiano) del otro en cuanto otro sujeto, pero tampoco tenemos un conocimiento inmediato y puro (en sentido cartesiano) de nosotros mismos. Desde esta perspectiva, el planteo empirista humeano invierte totalmente la asimetría cartesiana: la simetría se logra, no "elevando" el conocimiento del otro al nivel de la intuición cartesiana, sino "rebajando" el tipo de conocimiento que podemos tener de nosotros mismos.

La conclusión, el desengaño respecto de lo que podemos percibir en nosotros mismos, nos permite advertir que se trata, en gran parte, de las mismas cosas que (a través de la simpatía y otros mecanismos pasionales) percibimos en los demás. Para tener un contacto con el otro es indispensable el contexto intersubjetivo dentro del cual dicho contacto tiene lugar (y que nosotros contribuimos a dar forma), del mismo modo que para tener un contacto conmigo mismo (autoconocimiento) tampoco cabe aislarme de dicho marco, pues gran parte de lo que somos en cuanto sujetos lo debemos también al efecto que los otros tienen sobre nosotros.

Estas consideraciones apuntan inequívocamente al centro de la cuestión: el sofisticado giro anticartesiano que promulga Hume encuentra su formulación metateórica más álgida en lo que denominamos al inicio de este trabajo el giro o inversión de la relación dual teoría-praxis. ${ }^{22}$ Contra el cartesianismo, que ha dado clara primacía a la dimensión teorética de la subjetividad, relegando el ámbito práctico a un segundo lugar (casi derivado y, en última instancia, importante sólo porque resulta a la larga inevitable), en la filosofía de Hume parece concretarse un cambio de perspectiva. La dimensión práctica de nuestras vidas adquiere mayor relevancia: este ámbito de la naturaleza humana es el que se muestra más influyente y determinante. El conjunto, más bien heterogéneo, de ideas, creencias y convenciones que conforman nuestra vida obtiene su sentido y significado únicamente a partir de las prácticas que las instauran.

Aunque Hume no lo diga expresamente, esta inversión atraviesa o sobrevuela todo el Tratado, perfilando una clara alternativa que reivindica

${ }^{22}$ Muy pocos intérpretes de Hume han reparado en esta inversión de la que hablamos, y mucho menos aún han profundizado en ella (en sus fundamentos e implicancias filosóficos). Los comentarios sugerentes de G. Deleuze (1977) son una notable excepción.

Diánoia, vol. LIX, no. 72 (mayo de 2014). 
la importancia que las prácticas de la vida diaria tienen en la conformación de nociones que sólo en una segunda instancia podrán adquirir un uso teórico o especulativo (sea o no legítimo). De hecho, una de las dificultades que Hume advierte para su propia filosofía es determinar los criterios o estándares (teóricos y metodológicos) que le permitirían abstraer, en la medida de lo posible, determinados conceptos o nociones de sus contextos y usos habituales para ser utilizados legítimamente en otros contextos (filosóficos o científicos). Quizá el ejemplo emblemático se encuentre en el extenso y profundo tratamiento de la causalidad que Hume elabora especialmente en T1.3.

El razonamiento causal no adquiere su sentido a partir de la idea de conexión necesaria; estas secciones del Tratado, muy conocidas y comentadas, se encargan de mostrarnos que de hecho carecemos de una idea de conexión necesaria siempre que intentemos derivarla a partir de la impresión que tenemos de los objetos conectados causalmente. Sólo en el rodeo que Hume hace en su explicación, a través del fenómeno de la creencia que explica nuestras inferencias causales particulares, es que puede finalmente darle un sentido a nuestra idea de conexión necesaria: se trata de una impresión interna o secundaria, debida al hábito que contrae nuestra mente como efecto de la conjunción constante de ambos objetos. En otras palabras, no tenemos una comprensión, por así llamarla, "teórica" o "teorética" de la causalidad antes de hacer uso de ella, sino que esta noción que manejamos ordinariamente (en el mundo de la vida común, como suele decir Hume) sólo surge como consecuencia de nuestra práctica, i.e., de nuestro hábito de inferir la existencia de un objeto al percibir su acompañante habitual.

Ahora bien, cuando los metafísicos o los teólogos pretenden extrapolar esta noción de causalidad, eliminando dicho carácter pragmático y empleándola dentro de sus sistemas filosóficos o religiosos, ya como una forma de necesidad lógica o conceptual, ya para inferir las características de la providencia (es decir, sobre el mandato divino de un Dios sobre el cual poca evidencia empírica tenemos), en ese mismo instante se transforma en una noción ininteligible, en una palabra vacía de sentido, pues el único sentido inteligible para nosotros (la idea de conjunción constante empíricamente constatada y la determinación concomitante de la mente a esperar el acompañante habitual) le ha sido arrancado junto con el contexto pragmático en el cual entendíamos su aplicación.

La idea de justicia, por dar otro ejemplo, extrae su significado no de una pura relación entre ideas, sino de la práctica colectiva que da sentido a su instauración y su reglamentación dentro y en relación con

Diánoia, vol. LIX, no. 72 (mayo de 2014). 
un contexto específico. Únicamente después de ello esas ideas pueden adquirir una dimensión teórica, al ser utilizadas para un abordaje de segundo orden (filosófico o científico en general). Pero si dicha noción de justicia se abstrae de los contextos en los que ha sido conformada interpersonalmente y se intenta universalizarla (es decir, que valga para cualquier situación o contexto), como lo hicieron muchos teóricos del iusnaturalismo, pierde el sentido que originalmente tenía dentro del contexto práctico concreto en el cual (y como producto del cual) surgió.

Por ello, si al intentar utilizar, en un nivel teorético, conceptos que continuamente utilizamos en nuestra vida común y diaria, como los de justicia o causalidad, se les despoja de los sentidos y las referencias que poseen en la vida común, en la práctica que los ha instaurado, dichos conceptos devienen ininteligibles.

Ahora bien, esta dimensión práctica, que se muestra como ámbito principal de desenvolvimiento de la naturaleza humana, se inscribe y se despliega dentro del marco de una comunidad intersubjetiva. Del mismo modo que la dimensión práctica es anterior a la teórica, la dimensión social es anterior al individualismo teórico cartesiano. Todas las ideas, creencias, conocimientos, costumbres, tradiciones y convenciones que dan forma a la subjetividad (en su práctica diaria) son de carácter supraindividual, tienen un origen y un desarrollo intersubjetivo y temporal. Por ende, el vínculo social de los sujetos entre sí resulta el punto clave para comprender el giro anticartesiano que imprime Hume a su teoría de la subjetividad. Y, como vimos, la simpatía (mecanismo de comunicación de sentimientos) es el mecanismo clave para comprender este carácter ontológico de la naturaleza humana: la subjetividad es el producto o efecto de un conjunto más bien heterogéneo y variado de condiciones, algunas de las cuales (entre las más relevantes) son de orden intersubjetivo y pueden tener lugar sólo dentro de formas sofisticadas de socialización.

De nuevo, el mecanismo de la simpatía resulta estar convenientemente ubicado en el punto clave de esta explicación, exhibiendo un doble frente que lo transforma en una pieza clave dentro del andamiaje conceptual elaborado por Hume. Por un lado, desde la perspectiva individual, aporta a los sujetos pasiones y emociones que resultan determinantes para la conformación de la subjetividad. Pero, por otro lado, entabla un vínculo fundacional entre sujetos, hace posible (y explica) el desarrollo de un marco intersubjetivo sobre el cual, y gracias al cual, se forman los sujetos en cuanto miembros de una comunidad intersubjetiva que los determina recíprocamente. 
Retomando los interrogantes de la sección II, resulta esclarecedor poder responderlos desde la perspectiva humeana que ha abierto este trabajo. Recordémoslos: (a) ¿qué razones (o evidencias) tenemos para concluir que existen, de hecho, otros sujetos además de mí?; (b) ¿cuál (o cuáles) entre nuestras facultades es(son) la(s) que nos garantiza(n) un conocimiento del otro en cuanto alter ego?; (c) ¿qué tipo de conocimiento tenemos del otro en cuanto alter ego?

Lo que se sostuvo en este trabajo permite concluir que, en sentido estricto, no son "razones" o "evidencias" las que nos permiten "concluir" la existencia del alter ego, sino que se trata de mecanismos pasionales (instintivos, involuntarios, prerreflexivos) que nos "inclinan" a creer en dicha existencia, incluso antes de interrogarnos teóricamente respecto de ellas. Esta "inclinación" se muestra de manera más patente en el carácter eminentemente práctico de nuestra relación con los demás sujetos. En otras palabras, los otros sujetos no son una consecuencia de mi razonamiento, sino que están constantemente presentes ante mí como contexto indispensable dentro del cual y hacia el cual se dirigen constantemente mis acciones.

Por esa misma razón, no hay una "facultad", como la razón o la intuición, que nos proporcione un "conocimiento", sino que son simplemente sentimientos, determinadas pasiones y emociones, que hacen su aparición en la mente como efecto, principalmente, de mecanismos como la simpatía. Preguntar, por lo tanto, qué tipo de conocimiento tenemos de los otros, es equívoco, pues se trata de una propensión natural anterior a cualquier representación de la misma que podamos formar.

Esta caracterización aclara la relación anunciada, al iniciar el trabajo, entre esta perspectiva humeana y algunos desarrollos anticartesianos contemporáneos. Hablar de un conocimiento de los otros es concebir la relación con los otros como una relación esencialmente epistemológica. La filosofía humeana, como aquí se ha mostrado, permite elaborar un marco alternativo para concebir las relaciones de alteridad, un abordaje que no es primeramente teórico, sino práctico.

Además, las pasiones que sostienen nuestros vínculos intersubjetivos hacen posible un tipo especial de sentimientos que son los sentimientos morales. Éstos, privativos de los seres humanos, establecen una dimensión diferencial en las relaciones intersubjetivas. Aunque sería equívoco, por anacrónico, entablar un vínculo tan estrecho entre esta propuesta humeana y algunas críticas metateóricas al sujeto moderno

Diánoia, vol. LIX, no. 72 (mayo de 2014). 
(como aquéllas de E. Lévinas mencionadas al inicio de este trabajo), de todas formas podemos advertir que esta lectura hace de Hume un filósofo mucho más complejo e interesante, en vistas a debates contemporáneos, de lo que usualmente se lo considera.

\section{BIBLIOGRAFÍA}

Árdal, P., 1966, Passion and Value in Hume's Treatise, Edinburgh University Press, Edimburgo.

Baier, A.C., 1991, A Progress of Sentiments: Reflections on Hume's Treatise, Harvard University Press, Cambridge, Mass.

Baillie, J., 2000, Hume on Morality, Routledge, Londres.

Deleuze, G., 1977, Empirismo y subjetividad, trad. H. Acevedo, Gedisa, Barcelona.

Descartes, R., 2004a, Discurso del método, trad. R. Frondizi, Terramar, La Plata; 1a. ed., 1637.

— 2004b, Meditaciones metafísicas, trad. M. García Morente, Terramar, La Plata; 1a. ed., 1641.

Frazer, M.L., 2010, The Enlightenment of Sympathy. Justice and the Moral Sentiments in the Eighteenth Century and Today, Oxford University Press, Oxford.

Fogelin, R., 1985, Hume's Skepticism in the Treatise of Human Nature, Routledge and Kegan Paul, Londres.

Garrett, D., 1997, Cognition and Commitment in Hume's Philosophy, Oxford University Press, Oxford.

Gottlieb, E., 2007, Feeling British. Sympathy and National Identity in Scottish and English Writing, 1707-1832, Associated University Presses, Nueva Jersey.

Hume, D., 2000, Treatise of Human Nature, ed. D.F. Norton y M.J. Norton, Oxford University Press, Oxford; 1a. ed., 1739-1740.

Kemp Smith, N., 1941, The Philosophy of David Hume: Its Origins and Central Doctrines, Macmillan, Londres.

Lamb, J., 2009, The Evolution of Sympathy in the Long Eighteenth Century, Pickering and Chatto, Londres.

Martínez de Pisón, J., 1992, Justicia y orden político en Hume. Sus fundamentos filosóficos, Centro de Estudios Constitucionales, Madrid.

McIntyre, J., 2009, "Hume and the Problem of Personal Identity", en D.F. Norton y J. Taylor, The Cambridge Companion to Hume, Cambridge University Press, Cambridge, pp. 177-208.

Morgan, M.L., 2011, The Cambridge Introduction to Emmanuel Levinas, Cambridge University Press, Nueva York.

Noonan, H.W., 2007, Hume, Oneworld Publications, Oxford.

— 1999, Hume on Knowledge, Routledge, Nueva York/Londres.

Penelhum, T., 2000, Themes in Hume: The Self, the Will, Religion, Clarendon, Oxford. 
Peperzak, A., 1993, To the Other: An Introduction to the Philosophy of Emmanuel Levinas, Perdue, West Lafayette.

Pérez Quintana, A., 2008, "La crítica de Levinas a la filosofía trascendental moderna", en A. Alonso Martós (comp.), Emmanuel Lévinas. La filosofía como ética, Universidad de Valencia, Valencia, pp. 69-90.

Pitson, A.E., 2002, Hume's Philosophy of the Self, Routledge, Londres.

_, 1996 , "Sympathy and Other Selves", Hume Studies, vol. 22, pp. 255271.

Strawson, G., 2011, The Evident Connexion: Hume on Personal Identity, Oxford University Press, Oxford.

— 1989, The Secret Connexion: Causation, Realism and David Hume, Oxford University Press, Oxford.

Stroud, B., 1977, Hume, Routledge, Londres.

Waldow, A., 2009, David Hume and the Problem of Other Minds, Continuum International Publishing Group, Londres.

Wright, J.P., 1983, The Sceptical Realism of David Hume, Manchester University Press, Manchester.

Recibido el 22 de julio de 2013; aceptado el 30 de enero de 2014.

Diánoia, vol. LIX, no. 72 (mayo de 2014). 